Pacific Journal of Mathematics

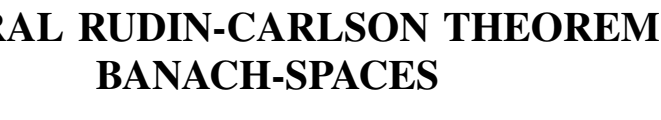




\title{
A GENERAL RUDIN-CARLSON THEOREM IN BANACH-SPACES
}

\author{
WALTER ROTH
}

Let $K$ be a closed subspace in a real or complex normed linear space $L$. The "Main Interpolation Problem" as formulated by $L$. Asimow reads as follows: Given a bounded convex neighborhood $V$ of 0 in $L$ and a bounded closed convex $U$ containing 0 , their polars $V^{0}$ and $U^{0}$ in the dual $L^{\prime}$ of $L$, define the functionals on $L p_{V_{K}}(x)=\sup \left(x, V^{0} \cap K^{0}\right)$ and $p_{V}(x)=\sup \left(x, U^{0}\right)$. For $x_{0} \in L$ we are looking for an element $x \in L$ satisfying

(1) $x-x_{0} \in K\left(\left.x\right|_{K^{0}}=\left.x_{0}\right|_{K^{0}}\right)$ and

(2) $p_{U}(x)=p_{V_{K}}\left(x_{0}\right)$ (exact solution), respectively

( $\left.2^{\prime}\right) \quad p_{U}(x) \leqq p_{V_{K}}\left(x_{0}\right)+\varepsilon$ for given $\varepsilon>0$ (approximate solution).

The problem is formulated in a different but equivalent way in this paper using the canonical projection $p$ from $L$ to $L / K$. For a real linear subspace $M$ of $L$, a convex cone $N$ in $M$ and bounded closed convex neighborhoods $U$ and $V$ we prove conditions in terms of the dual space of $L$ which are necessary and sufficient for the inclusions

$$
p(N \cap U) \supset p(M) \cap \underline{p(V)} \operatorname{resp} . p(N \cap U) \supset p(M) \cap \overline{p(V)}
$$

( $\{\cdots\}$ means the topological interior, $\{\cdots\}$, the closure).

Theorem 1 shows the equivalence of the first inclusion to the existence of a not necessarily linear map with certain properties form the dual $L^{\prime}$ to $K^{0}$, the second inclusion is shown to be valid if the first one holds for a certain family of 0 -neighborhoods $U$ and $V$. Theorems 2 and 3 are applications of the first one and in the case $L=C(X)$, where $X$ is a compact Hausdorff space give generalizations of several well-known results: Gamelin's extended RudinCarleson theorem [12], theorems by Björk [10] and Alfsen [1] and T.B. Andersen's split-face theorem [3]. Some of the following results are closely related to Ando's paper [4] on closed range theorems, which gives conditions for the validity of the second inclusion if there exists a projection in the dual of $L$ with range $K^{0}$. The notation of "splitability" there coincides with restrictions on neighborhoods ("strongly admissible") in this paper.

I am grateful to L. Asimow for some useful suggestions on the subject.

1. A basic theorem. Let $L$ be a real or complex normed linear space, $L^{\prime}$ its dual. The polar $S^{0}$ of a subset $S$ in $L$ is defined as the 
set of all $\mu \in L^{\prime}$ such that $\operatorname{Re} \mu(f) \leqq 1$ for every $f \in S$. The following well-known facts on polars are used in this paper (for proofs, for instance see [17]): The bipolar of $S$ in $L$ is the $\sigma\left(L, L^{\prime}\right)$ closed convex hull of $S \cup\{0\}$. If $S_{1}, S_{2}$ are subsets of $L$, we have $\left(S_{1} \cup S_{2}\right)^{0}=$ $S_{1}^{0} \cap S_{2}^{0}$. If both $S_{1}$ and $S_{2}$ are closed and convex $\left(S_{1} \cup S_{2}\right)^{0}$ coinsides with the $\sigma\left(L^{\prime}, L\right)$ closure of the convex hull of $S_{1}^{0} \cup S_{2}^{0}$ in $L^{\prime}$, and if in addition $S_{1}$ and $S_{2}$ are 0 -neighborhoods in $L$ this convex hull is $\sigma\left(L^{\prime}, L\right)$ compact, hence $\left(S_{1} \cap S_{2}\right)^{0}=\operatorname{conv}\left(S_{1}^{0} \cup S_{2}^{0}\right)$. We state now our first theorem.

THEOREM 1. Let $K$ be a closed subspace of the real or complex normed linear space $L, M$ a real linear subspace of $L, N$ a norm complete convex cone in $M, V$ a bounded convex, $U$ a bounded convex and closed neighborhood of 0 in $L . \quad p: L \rightarrow L / K$ is the canonical projection. For the following assertions
(a) $p(N \cap U) \supset p(M) \cap \underline{p(V)}$.

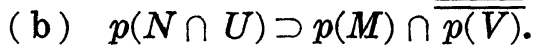
(c) $\{p(N \cap U)\}^{0} \subset\{p(M) \cap p(V)\}^{0}$.
(d) There is a map $\varphi: L^{\prime} \rightarrow K^{0}$ with the properties:

(d1) For every $\mu \in K^{0}(\varphi(\mu)-\mu) \in M^{0}$.

(d2) For every $\mu \in U^{0}$ and every $f \in M$ such that $p(f) \in p(V)$ we have $\operatorname{Re} \varphi(\mu)(f) \leqq 1$.

(d3) For all $\mu, \nu \in L^{\prime}$ such that $(\mu-\nu) \in N^{0}$ we have $(\varphi(\mu)-\varphi(\nu)) \in M^{0}$.

(e) For every $h \in N \cap \underline{U}$ such that the Minkowski functional of $K+V q_{K+V}(h)<1$ define

$$
U_{h}=U \cap \frac{1}{\lambda(h)}(U-h), \quad \lambda(h)=1-q_{K+V}(h)
$$

and we have

$$
p\left(N \cap U_{h}\right) \supset p(M) \cap \underline{p(V)} .
$$

(a) and (c) are equivalent, (d) implies (a), (a) implies (d) if $N$ is a real linear space, (e) implies (b), and (b) implies (a).

Proof. The implications $(\mathrm{a}) \Rightarrow(\mathrm{c})$ and $(\mathrm{b}) \Rightarrow(\mathrm{a})$ are trivial. To prove $(\mathrm{c}) \Rightarrow$ (a) and $(\mathrm{e}) \Rightarrow(\mathrm{b})$ we need Lemma 1 .

$(c) \Rightarrow(a)$ : Taking the polars on both sides of inclusion (c) shows that $\overline{p(N \cap U)} \supset p(M) \cap p(V)$. Applying Lemma 1, part (1), with $A=L, B=p(M) \subset L / K, C=N \cap U$ and $D=p(M) \cap p(V)$ we conclude (a).

(e) $\Rightarrow(b)$ is a consequence of Lemma 1 , part (2) with the same insertion for $A, B, C$ and $D$. Then $U \cap 1 / \lambda(C-h)=U \cap 1 / \lambda(N \cap U-h)=$ 
$U \cap 1 / \lambda(N-h \cap U-h) \supset U \cap N \cap 1 / \lambda(U-h)=N \cap U_{h}$. Obviously for $h \in N \cap U$ both Minkowski-functionals in (e) and in Lemma 1 are equal:

$$
\begin{aligned}
q_{0}(p(h)) & =\inf \{\rho \mid p(h) \in \rho(p(M) \cap p(V))\} \\
& =\inf \{\rho \mid h \in \rho(M+K \cap V+K)\} \\
& =\inf \{\rho \mid h \in \rho(V+K)\}=q_{V+K}(h) .
\end{aligned}
$$

So (e) implies the assumptions of Lemma 1, part (2), and we derive (b).

$(d) \Rightarrow(c)$ : This argument makes use of an extended Hahn-Banach theorem by Kaufmann [15] which states the following:

Let $L$ be a real linear space, $N$ a convex cone in $L, q$ a subadditive, positive-homogeneous functional on $L$, and let $\mu$ be an additive positive-homogeneous functional on $N$ such that $\mu \leqq q$ on $N$. Then there is a linear functional $\theta$ on $L$ such that $\theta \leqq q$ and $\mu \leqq \theta$ on $N$.

Now suppose (d) holds and let $\mu$ be an element of $(p(N \cap U))^{\circ}$. Then $\mu \in K^{0}\left(K^{0}\right.$ is the dual of $\left.L / K\right)$ and $\operatorname{Re} \mu(f) \leqq 1$ for every $f \in N \cap U$. Let $q$ be the positive-homogeneous subadditive functional on $L$ generated by $U$ :

$$
q(f)=\inf \left\{\lambda \in \boldsymbol{R}_{+} \mid f \in \lambda U\right\} .
$$

There is a constant $r>0$ such that $q(f) \leqq r\|f\|$ for every $f$ in $L$, because $U$ is a neighborhood of 0 .

Let $\mu_{1}$ be the real functional on $L: \mu_{1}=\operatorname{Re} \mu$. Then $\mu_{1}(f) \leqq q(f)$ for every $f$ in $N$ and applying Kaufmann's theorem we find a real valued functional $\mu_{2}$ on $L$ such that

$$
\mu_{2}(f) \leqq q(f) \text { for } f \in L \text { and } \mu_{1}(f) \leqq \mu_{2}(f) \text { on } N \text {. }
$$

Clearly $\mu_{2}$ is continuous, hence the real part of an element $\mu_{3} \in L^{\prime}$. So we have for every $f$ in $M$

$$
\operatorname{Re} \varphi\left(\mu_{3}\right)(f)=\operatorname{Re} \varphi(\mu)(f)=\operatorname{Re} \mu(f) .
$$

(This is a consequence of assumption (d3) because $\mu-\mu_{3} \in N^{0}$, hence $\phi(\mu)-\varphi\left(\mu_{3}\right) \in M^{0}$, and of (d1) because $\mu \in K^{0}$, hence $\phi(\mu)-\phi \in M^{0}$.) Now suppose $f \in M$ such that $p(f) \in p(V)$, then (d2) implies $\operatorname{Re} \varphi\left(\mu_{3}\right)(f) \leqq 1$, because $\mu_{3} \in U^{0}$. Therefore $\operatorname{Re} \mu(f) \leqq 1$, and $\mu$ belongs to the polar of $p(M) \cap p(V)$.

If $N$ is a real linear space too we prove the implication

(a) $\Rightarrow(\mathrm{d})$ : Suppose $p(N \cap U) \supset p(M) \cap \underline{p(V)}$ and define the map $\varphi: L^{\prime} \rightarrow K^{0}$ using the axiom of choice as follows: 


$$
\varphi(\mu)=\left\{\begin{array}{l}
\bar{\mu} \text { if there is } \bar{\mu} \in K^{0} \text { such that } \mu-\bar{\mu} \in N^{0} \\
0, \text { else } .
\end{array}\right.
$$

Thus $\varphi$ is well-defined and meets the requirements (d1), (d2), (d3):

(d1) Suppose $\mu \in K^{0}$ and $\bar{\mu} \in K^{0}$ such that $\mu-\bar{\mu} \in N^{0}$.

For every $f \in M$ there exists by assumption $g \in N$ such that $p(f)=p(g)$, hence $\operatorname{Re} \mu(f)=\operatorname{Re} \mu(g)=\operatorname{Re} \bar{\mu}(g)=\operatorname{Re} \varphi(\mu)(f)$.

(d2) Suppose $\mu \in U^{0}, \bar{\mu}=\phi(\mu), f \in M$ such that $p(f) \in p(V)$. Then for every $\gamma \in(0,1) \gamma f \in p(M) \cap \underline{p(V)}$, therefore we find $g \in N \cap U$ with $p(g)=p(\gamma f)$, hence $\operatorname{Re} \overline{\varphi(\mu)(f)}=\operatorname{Re} \varphi(\mu)((1 / \gamma) g)=$ $\operatorname{Re} \bar{\mu}((1 / \gamma) g)=\operatorname{Re} \mu((1 / \gamma) g) \leqq(1 / \gamma)$. Thus $\operatorname{Re} \phi(\mu)(f) \leqq 1$.

(d3) Suppose $\mu, \nu \in L^{\prime}$ such that $(\mu-\nu) \in N^{0}$.

Then in case there is no proper $\bar{\mu}$ in $K^{0}$, we have $\varphi(\mu)=\varphi(\nu)=0$. Else let be $\bar{\mu}=\varphi(\mu), \bar{\nu}=\varphi(\nu)$. Then $\bar{\mu}-\mu \in N^{0}, \bar{\nu}-\nu \in N^{0}$, hence $\bar{\mu}-\bar{\nu} \in N^{0}$, and $\bar{\mu}-\bar{\nu} \in M^{0}$ as well because $\bar{\mu}-\bar{\nu} \in K^{0}$.

To complete the proof of Theorem 1 we need the following lemma:

Lemma 1. Let $A$ and $B$ be normed real linear spaces, $p: A \rightarrow B$ a continuous linear map, $C$ a complete bounded convex subset in $A$ containing $0, D$ a bounded convex neighborhood of 0 in $B$. Then

(1) $\overline{p(C)} \supset D$ implies $p(C) \cap \underline{D}$.

(2) If there is a bounded neighborhood $U$ of 0 in $A$ containing $C$, such that for every $h$ in the algebraic interior of $C$ for which

$$
\lambda(h)=\sup \left\{\rho \in R_{+} \mid p(h) \in(1-\rho) D\right\}=1-q_{D}(p(h))>0
$$

(where $q_{D}$ denotes the Minkowski-functional of $D$ on $B$ )

$$
\overline{p\left(U \cap \frac{1}{\lambda(h)}(C-h)\right)} \supset D,
$$

then $p(C) \supset \bar{D}$.

Proof. (1) Suppose $\overline{p(C)} \supset D$ and let $f \in D$. Given $\varepsilon>0$ there is $g_{0} \in C$ such that $\left\|f-p\left(g_{0}\right)\right\|<\varepsilon$. Suppose $g_{1}, \cdots, g_{n} \in C$ have been selected such that

$$
\left\|f-p\left(\sum_{i=0}^{k}\left(\frac{\varepsilon}{r}\right)^{i} g_{i}\right)\right\| \leqq \varepsilon\left(\frac{\varepsilon}{r}\right)^{k}, \quad \text { for every } \quad k=1, \cdots, n
$$

where $r>0$ is a constant, such that $r E_{B} \subset D . \quad\left(E_{B}\right.$ denotes the closed unit ball in $B$.) Then $(r / \varepsilon)^{n+1}\left(f-p\left(\sum_{i=0}^{n}(\varepsilon / r)^{i} g_{i}\right)\right) \in D$ and we find $g_{n+1} \in C$ such that

$$
\left\|\left(\frac{r}{\varepsilon}\right)^{n+1}\left(f-p\left(\sum_{i=0}^{n}\left(\frac{\varepsilon}{r}\right)^{i} g_{i}\right)\right)-p\left(g_{n+1}\right)\right\| \leqq \varepsilon,
$$


hence

$$
\left\|f-p\left(\sum_{i=0}^{n+1}\left(\frac{\varepsilon}{r}\right)^{i} g_{i}\right)\right\| \leqq \varepsilon\left(\frac{\varepsilon}{r}\right)^{n+1} \text {. }
$$

Set $g=\sum_{i=0}^{\infty}(\varepsilon / r)^{i} g_{i}$. Then $p(g)=f$, and $g \in(1 /(1-(\varepsilon / r))) C$, hence for every $\gamma>1, p(\gamma C) \supset D, p(C) \supset(1 / \gamma) D$, which proves part (1).

(2) $U$ is bounded, so $U \subset R E_{A}$, where $E_{A}$ denotes the unit ball in $A$. Let $f \in \bar{D}$. Then $(f / 2) \in \underline{D}\left(1-(1 / 2)^{2}\right)$ and by hypothesis (set $h=0)$ and part (1) there is $g_{1} \in C\left(1-(1 / 2)^{2}\right)$ such that $p\left(g_{1}\right)=(f / 2)$ and $\left\|g_{1}\right\| \leqq\left(3 / 2^{2}\right) R$ (because $\left.g_{1} \in\left(1-(1 / 2)^{2}\right) U\right)$. Suppose $g_{1}, g_{2}, \cdots, g_{n}$ have been selected such that $\sum_{i=1}^{n} g_{i} \in C\left(1-(1 / 2)^{n+1}\right), p\left(g_{i}\right)=\left(f_{1} / 2^{i}\right)$, $\left\|g_{i}\right\| \leqq\left(3 / 2^{i+1}\right) R, i=1, \cdots, n$. Set

$$
h=\frac{1}{\left(1-\left(\frac{1}{2}\right)^{n+2}\right)} \sum_{i=1}^{n} g_{i} .
$$

Then $h \in \mathbf{U}_{0 \leqq r<1} \gamma C$ and

$$
\begin{aligned}
p(h) & =\frac{1}{\left(1-\left(\frac{1}{2}\right)^{n+2}\right)} \sum_{i=1}^{n} \frac{f}{2^{i}}=\frac{\left(1-\left(\frac{1}{2}\right)^{n}\right)}{\left(1-\left(\frac{1}{2}\right)^{n+2}\right)} f \\
& =\frac{2^{n+2}-2^{2}}{2^{n+2}-1} f \in \frac{2^{n+2}-2^{2}}{2^{n+2}-1} D,
\end{aligned}
$$

hence

$$
\lambda(h) \geqq 1-\frac{2^{n+2}-2^{2}}{2^{n+2}-1}=\frac{3}{2^{n+2}-1} .
$$

By hypothesis and part (1) of the lemma then

$$
p\left(U \cap \frac{2^{n+2}-1}{3}(C-h)\right) \supset \underline{D},
$$

and there is

$$
g^{\prime} \in U \cap \frac{2^{n+2}-1}{3}(C-h)
$$

such that

$$
p\left(g^{\prime}\right)=\frac{2}{3} f
$$

Now let 


$$
g_{n+1}=\frac{3}{2} \frac{1}{2^{n+1}} g^{\prime}
$$

Then

$$
\begin{aligned}
g_{n+1} & \in \frac{3}{2} \frac{1}{2^{n+1}} \cdot \frac{2^{n+2}-1}{3}(C-h)=\left(1-\left(\frac{1}{2}\right)^{n+2}\right)(C-h) \\
& =\left(1-\left(\frac{1}{2}\right)\right)\left(C-\frac{1}{\left(1-\left(\frac{1}{2}\right)^{n+2}\right)} \sum_{i=1}^{n} g_{i}\right)=\left(1-\left(\frac{1}{2}\right)^{n+2}\right) C-\sum_{i=1}^{n} g_{i},
\end{aligned}
$$

hence

$$
\sum_{i=1}^{n+1} g_{i} \in C\left(1-\left(\frac{1}{2}\right)^{n+2}\right), \quad p\left(g_{n+1}\right)=\frac{f}{2^{n+1}}, \quad\left\|g_{n+1}\right\| \leqq \frac{3}{2^{n+2}} R .
$$

Set $g=\sum_{i=1}^{\infty} g_{i}$. Then $p(g)=f$ and $g \in C$, which completes the proof.

2. A Rudin-Carleson theorem. Throughout this section we assume that $K^{0}$ is the range of a norm continuous linear projection $\pi$ in the dual space $L^{\prime}$ of $L$. Applying the implications (d) $\Rightarrow($ a) (setting $\varphi=\pi$ ) and $(e) \Rightarrow(b)$ in Theorem 1 we derive an extended Rudin-Carleson-type theorem in Banach spaces. Since the above assumption coincides with Ando's [4] some of the results are related to his.

Let $K$ be a closed subspace of the Banach space $L, \pi: L^{\prime} \rightarrow K^{0}$ a continuous linear projection. To apply Theorem 1 we need some requirements on "admissible" neighborhoods of the origin in $L$.

Definition. Let $U$ and $V$ be closed convex bounded neighborhoods of 0 in $L . \quad(U, V)$ is called admissible, iff $\pi\left(U^{0}\right) \subset V^{0} . U$ is called strongly admissible, iff $U^{0}=\overline{\operatorname{conv}\left\{\pi\left(U^{0}\right) \cup(I-\pi)\left(U^{0}\right)\right\}}(\overline{\{\}}$ denotes the closure in the norm topology of $L^{\prime}$.)

REMARKS.

(2.1) $(E,(1 /\|\pi\|) E)$, where $E$ is the closed unit ball in $L$, is admissible.

(2.2) If $L$ is an $A M$-space (Banach lattice with property $\|f \vee g\|=$ $\|f\| \vee\|g\|$ for all positive elements $f, g$ in $L$, cf. [19]), $K$ an ideal in $L, \pi: L^{\prime} \rightarrow K^{0}$ the band projection, then the closed unit ball $E$ in $L$ is strongly admissible: The inclusion $E^{0} \supset \overline{\operatorname{conv}\left(\pi\left(E^{0}\right) \cup(I-\pi)\left(E^{0}\right)\right)}$ is trivial. Conversely let $\mu \in E^{0}$, then $\mu=\pi(\mu)+(I-\pi) \mu$, and because $\pi(\mu)$ and $(I-\pi) \mu$ are orthogonal and $L^{\prime}$ is an $A L$-space $\|\pi(\mu)||+||(I-\pi) \mu||=|||\pi(\mu)|+|(I-\pi)(\mu)||| \leqq\| \pi(|\mu|)+(I-\pi)(|\mu|)|| \leqq 1$, hence $\mu \in \operatorname{conv}\left(\pi\left(E^{0}\right) \cup(I-\pi)\left(E^{0}\right)\right)$. 
(2.3) Let $\left(U_{1}, V_{1}\right)$ and $\left(U_{2}, V_{2}\right)$ be admissible 0-neighborhoods. Then $\left(U_{1} \cap U_{2}, V_{1} \cap V_{2}\right)$ is admissible.

This is an immediate consequence of the fact that $\left(U_{1} \cap U_{2}\right)^{0}=$ $\operatorname{conv}\left(U_{1}^{0} \cup U_{2}^{0}\right)$, hence $\pi\left(\left(U_{1} \cap U_{2}\right)^{0}\right)=\pi\left(\operatorname{conv}\left(U_{1}^{0} \cup U_{2}^{0}\right) \subset \operatorname{conv}\left(\pi\left(U_{1}^{0}\right) \cup\right.\right.$ $\left.\left.\pi\left(U_{2}^{0}\right)\right) \subset \operatorname{conv}\left(V_{1}^{0} \cup V_{2}^{0}\right)=\left(V_{1} \cap V_{2}\right)^{0}\right)$.

(2.4) If both $U$ and $V$ are strongly admissible, $U \cap V$ is strongly admissible, because

$$
\begin{aligned}
(U \cap V)^{0} & =\operatorname{conv}\left(U^{0} \cup V^{0}\right) \\
& \left.=\operatorname{conv} \overline{\left(\operatorname{conv}\left(\pi\left(U^{0}\right)\right) \cup(I-\pi)\left(U^{0}\right)\right) \cup \operatorname{conv}\left(\pi\left(V^{0}\right) \cup(I-\pi)\left(V^{0}\right)\right.}\right) \\
& =\overline{\operatorname{conv}\left(\pi\left(U^{0}\right) \cup \pi\left(V^{0}\right) \cup(I-\pi)\left(U^{0}\right) \cup(I-\pi)\left(V^{0}\right)\right)} \\
& =\operatorname{conv(\pi (\operatorname {conv}(U^{0}\cup V^{0})\cup (I-\pi )(\operatorname {conv}(U^{0}\cap V^{0})))} \\
& =\operatorname{conv~(\pi (U\cap V)^{0}\cup (I-\pi )(U\cap V)^{0})} .
\end{aligned}
$$

(2.5) Let $U$ be strongly admissible, $h \in \underline{U}$. Then $\left(U_{h}, U\right)$ is admissible $\left(U_{h}\right.$ was defined: $\left.U_{h}=U \cap(1 / \lambda(h))(U-h)\right)$. To prove (2.5) it is sufficient (because of (2.3)) to show that $((1 / \lambda(h))(U-h), U)$ is admissible, i.e., $\pi((1 / \lambda(h))(U-h))^{0} \subset U^{0}$, i.e., $\pi(U-h)^{0} \subset(1 / \lambda(h)) U^{0}$. Let $\mu \in(U-h)^{0}$, then $\operatorname{Re} \mu(f) \leqq 1+\operatorname{Re} \mu(h)$ for every $f \in U$. From $0 \in \underline{U}$ we conclude that $\operatorname{Re} \mu(h)>-1$, hence $\mu \in(1+\operatorname{Re} \mu(h)) U^{0}$. By assumption $U$ is strongly admissible, hence there is $\nu \in L^{\prime}$ such that $\|\mu-\nu\|<\varepsilon, \nu=\lambda_{1} \nu_{1}+\lambda_{2} \nu_{2}, \lambda_{1}+\lambda_{2}=1, \lambda_{1}, \lambda_{2} \geqq 0, \nu_{1} \in(1+\operatorname{Re}(h)) \pi\left(U^{0}\right)$, $\nu_{2} \in(1+\operatorname{Re} \mu(h))\left(U^{0}\right)$. Then $\pi(\nu)=\lambda_{1} \nu_{1},(I-\pi)(\nu)=\lambda_{2} \nu_{2}$.

From the definition of $\lambda(h)=\sup \left\{\rho \in R_{+} \mid p(h) \in(1-\rho) p(U)\right\}$ and because $\pi(\nu) \in K^{0}$ for every $f \in U$ we conclude $\operatorname{Re} \pi(\nu)(h)+\lambda \operatorname{Re} \pi(\nu)(f) \leqq$ $\sup \{\pi(\nu)(g) \mid g \in U\} \leqq \lambda_{1}(1+\operatorname{Re} \mu(h))$, hence $\lambda \operatorname{Re} \pi(\nu)(f) \leqq \lambda_{1}(1+\operatorname{Re} \mu(h))+$ $\operatorname{Re}(I-\pi)(\nu)(h)-\operatorname{Re} \nu(h) \leqq \lambda_{1}(1+\operatorname{Re} \mu(h))+\lambda_{2}(1+\operatorname{Re} \mu(h))-\operatorname{Re} \nu(h)=$ $1+\operatorname{Re} \mu(h)-\operatorname{Re} \nu(h) \leqq 1+\varepsilon\|h\|$. (Note that $h \in \underline{U},(I-\pi)(\nu) \in \lambda_{2}(1+$ $\operatorname{Re} \mu(h))(I-\pi)\left(U^{0}\right)$ imply $\operatorname{Re}(I-\pi)(\nu)(h) \leqq \lambda_{9}(1+\operatorname{Re} \mu(h))$. $)$

Thus $\pi(\nu) \in(1+\varepsilon\|h\|)\left(U^{0} / \lambda\right)$ for $\varepsilon>0$. Because $\pi$ is norm continuous from this we conclude $\pi(\mu) \in\left(U^{0} / \lambda\right)=\left(\overline{U^{0}} / \lambda\right)$.

Now Theorem 2 is at hand.

THEOREM 2. Let $K$ be a closed subspace of the real or complex Banach space $L, K^{0}$ be the range of a norm continuous linear projection $\pi$ on $L^{\prime}, p: L \rightarrow L / K$ the canonical map. Suppose $M$ is a real linear subspace of $L, N$ a norm closed convex cone in $M$. For the following assertions

(a) For all closed convex bounded neighborhoods $U$ and $V$ of $O$ in $L$ such that $(U, V)$ is admissible

$$
p(N \cap U) \supset p(M) \cap \underline{p(V)} .
$$

(b) For every strongly admissible closed convex bounded neigh- 
borhood $U$ of 0 in $L$

$$
p(N \cap U) \supset P(M) \cap \overline{p(U)} .
$$

( c ) $\pi\left(N^{0}\right) \subset M^{0}$.

(a) implies (b), and (c) and (a) are equivalent.

Proof.

(a) $\Rightarrow(b)$ is an immediate consequence of implication

$(e) \Rightarrow(b)$ in Theorem 1 and of Remark (2.5). To prove

(c) $\Rightarrow$ (a) we show Condition (d) in Theorem 1 holds with $\varphi=\pi$.

(d1) is trivial, and because of the linearity of $\pi$ (d3) corresponds to assertion (c) of Theorem 2. To verify (d2) let $\mu \in U^{0}, f \in V$. Then $\pi(\mu) \in V^{0}$ because $(U, V)$ is admissible, hence $\operatorname{Re} \pi(\mu)(f) \leqq 1$.

(a) $\Rightarrow$ (c). Assume (a) holds and let $\mu \in N^{0}, f \in M$. To prove $\operatorname{Re} \pi(\mu)(f)=0$ we have to define proper 0 -neighborhoods $U$ and $V$. Let

$$
V=(1 /\|\pi\|) E \text { and } U_{\varepsilon}=E \cap\{h \in L||(I-\pi)(\mu)(h) \mid \leqq \varepsilon\}
$$

where $E$ denotes the closed unit ball in $L$. Both $U_{\varepsilon}$ and $V$ are bounded convex and closed and $\left(U_{\varepsilon}, V\right)$ is admissible: $U_{\varepsilon}^{0}=$ $\operatorname{conv}\left(E^{0} \cup\{\cdots\}^{0}\right)$, hence $\pi\left(U_{\varepsilon}^{0}\right) \subset \operatorname{conv}\left(\pi\left(E^{0}\right) \cup \pi\{\cdots\}^{0}\right)$. So obviously it suffices to verify $\pi\{\cdots\}^{0} \subset V^{0}$. But $\{\cdots\}=\varepsilon \cdot\left\{e^{i \alpha}(I-\pi)(\mu) \mid \alpha \in[0,2 \pi]\right\}^{0}$, therefore $\{\cdots\}^{0}=(1 / \varepsilon)\{\lambda(I-\pi)(\mu)|| \lambda \mid \leqq 1\}$ and $\pi\{\cdots\}^{0}=\{0\}$.

Now select $\lambda>\|f\| \cdot\|\pi\|$. Then $(1 / \lambda) f \in \underline{V}$ and $p((1 / \lambda) f) \in p(M) \cap$ $\underline{p(V)}$ and by assumption there is $g_{\varepsilon} \in N \cap U_{\varepsilon}$ such that $p\left(g_{\varepsilon}\right)=p((1 / \lambda) f)$, hence $\left|(I-\pi)(\mu)\left(g_{\varepsilon}\right)\right| \leqq \varepsilon$, i.e., $\left|\mu\left(g_{\varepsilon}\right)-\pi(\mu)\left(g_{\varepsilon}\right)\right| \leqq \varepsilon$.

On the other hand we know because $g_{\varepsilon}-(1 / \lambda) f \in K, \mu \in N^{0}$ and $\pi(\mu) \in K^{0}$ that $\operatorname{Re} \pi(\mu)((1 / \lambda) f)=\operatorname{Re} \pi(\mu)\left(g_{\varepsilon}\right) \leqq \operatorname{Re} \mu\left(g_{\varepsilon}\right)+\varepsilon \leqq \varepsilon$.

The argument holds for every $\varepsilon>0$ independent of $\lambda$, hence $\operatorname{Re} \pi(\mu)(f / \lambda) \leqq 0$ and $\pi(\mu) \in M^{0}$.

3. Applications in Banach lattices. In this section we are going to take advantage of the fact that the map $\varphi: L^{\prime} \rightarrow K^{0}$ in Theorem (1d) needs not necessarily be linear. For the following suppose $L$ is a real or complex Banach lattice, i.e., in the complex case $L$ is the complexification of a real Banach lattice $L_{0}$ (for details cf. [19]) $L=L_{0}+i L_{0}$. Let $K$ be an ideal in $L$, then $K^{0}$ is a band in the order complete dual $L^{\prime}=L_{0}^{\prime}+i L_{0}^{\prime}$ of $L$. By $\pi$ we denote the band projection from $L^{\prime}$ onto $K^{0}$. $\pi$ is norm continuous and monotone (cf. ([19]). As before $M$ is a real linear subspace of $L, N$ a closed convex cone in $M$. For the construction of $\varphi$ we introduce a new parameter: 
Let $R$ be a sup-stable (i.e., $f \vee g \in R$ for all $f, g \in R$ ) convex cone in $L_{0}$ such that

(3.1) $\operatorname{Re}(\operatorname{lin} N) \subset R$, i.e., $R$ contains all real parts of the elements of lin $N$, the complex linear hull of $N$.

(3.2) $R$ is total in $L_{0}$, i.e., $\overline{R-R}=L_{0}$.

(3.3) $\left(E_{L_{0}} \cap R\right)+L_{+}$, where $E_{L_{0}}$ denotes the unit ball, $L_{+}$the positive cone in $L_{0}$, is a neighborhood of the origin in $L_{0}$.

In straightforward analogy to the concept of the Choquet ordering for measures on a convex compact Hausdorff space we define an order relation " $<_{R}$ " on $L_{+}^{\prime}\left(L_{+}^{\prime}\right.$ denotes the positive cone in $\left.L_{0}^{\prime}\right)$ by $\mu<_{R} \nu$ iff $\mu(f) \leqq \nu(f)$ for all $f \in R$. Here $R$ takes the part of the continuous convex functions in the classical case (cf. Alfsen [1]). Like there we show that there are sufficiently many maximal elements in this ordering (Lemma 2) and then define $\varphi$ using the axiom of choice of the composition of a map from $L^{\prime}$ in the set of maximal elements and the band projection onto $K^{0}$. According to the choice of the parameter $R$ Theorem 3 yields a wide range of applications. For $R=L_{0}$ for instance, the ordering is trivial and it leads to the Rudin-Carleson-type theorem of $\S 2$. In $\S 4$ we shall apply it to the case $L=C(X)$ with different choices for $R$.

The proof of Lemma 4.1 in [16] can be adapted to derive the following lemma on the existence of maximal elements in $L_{+}^{\prime}$. (Note that condition (3.3) for $R$ guarantees the $\sigma\left(L^{\prime}, L\right)$ compactness of the set $\left\{\nu \in L_{+}^{\prime} \mid \nu>_{R} \mu\right\}$ for given $\mu \in L_{+}^{\prime}$.)

Lemma 2. For every $\mu \in L_{+}^{\prime}$ there is $\bar{\mu} \in L_{+}^{\prime}$ such that $\bar{\mu}>_{R} \mu$ and $\bar{\mu}$ is maximal in the ordering "> $>_{R}$.

For every $f \in L_{0}$ define the upper respectively lower $R$-envelope (cf. [1], §5) in $L_{0}^{\prime \prime}$, the order complete bidual of $L_{0}$

$$
\hat{f}=\inf \{h \in-R \mid h \geqq f\}, \quad \check{f}=\sup \{h \in R \mid h \leqq f\} .
$$

Then for $\mu, \nu \in L_{+}^{\prime} \mu<_{R} \nu$ implies $\mu(\check{f}) \leqq \nu(\check{f})$ and $\mu(\hat{f}) \geqq \nu(\hat{f})$. This is an immediate consequence of Propositions 4.2 and 4.5 in Schäfer's book [19], because $\mu(\hat{f})=\inf \{\mu(h) \mid h \in-R, h \geqq f\}$ for positive $\mu$.

Corresponding to the set of boundary measures in Choquet-theory we define

$$
\partial L^{\prime}=\left\{\mu \in L^{\prime}|| \mu \mid \text { is } R \text {-maximal }\right\} \text {. }
$$

(Recall that $|\mu|=\sup _{\alpha \in[0,2 \pi]}\left|\cos \alpha \mu_{1}+\sin \alpha \mu_{2}\right| \in L_{0}^{\prime}$ where $\mu=\mu_{1}+i \mu_{2}$.)

For every $\mu \in L^{\prime}$ there is $\bar{\mu} \in \partial L^{\prime}$ such that $\mu-\bar{\mu} \in(\text { lin } N)^{0}$, because there is a decomposition of $\mu=\left(\mu_{1}-\mu_{2}\right)+i\left(\mu_{3}-\mu_{4}\right)$ such that 
$\mu_{i} \in L_{+}^{\prime}$. According to Lemma 2 select $\bar{\mu}_{i} \in L_{+}^{\prime}$ such that the $\bar{\mu}_{i}$ are $R$-maximal and $\bar{\mu}_{i}>_{R} \mu_{i}$, hence $\bar{\mu}_{i}-\mu_{i} \in(\operatorname{lin} N)^{0}$. Set $\bar{\mu}=\left(\bar{\mu}_{1}-\bar{\mu}_{2}\right)+$ $i\left(\bar{\mu}_{3}-\bar{\mu}_{4}\right)$.

A very useful characterization of the elements of $\partial L^{\prime}$ is given by a reformulation of [1], Proposition I.4.5.

LEMMA 3. $\mu \in \partial L^{\prime}$ if and only if $|\mu|(f)=|\mu|(\hat{f})$ for every $f \in L_{0}$. $\partial L^{\prime}$ is an order ideal in $L^{\prime}$.

The proof of the first assertion follows straightforward the proof of Proposition I.3.5 and the argument in Proposition I.4.5 in Alfsen's book [1]. To verify that $\partial L^{\prime}$ is an order ideal in $L^{\prime}$ let $\mu, \nu \in \partial L^{\prime}$. Then for $f \in L_{0} \hat{f}-f$ is clearly positive in $L^{\prime \prime},|\mu+\nu|(\hat{f}-f) \leqq|\mu|(\hat{f}-f)+$ $|\nu|(\hat{f}-f)=0$, hence $\mu+\nu \in \partial L^{\prime}$. If $\mu \in \partial L^{\prime}$ and $\nu \in L^{\prime}$ such that $|\boldsymbol{\nu}| \leqq|\boldsymbol{\mu}|$. Then $|\boldsymbol{\nu}|(\hat{f}-f) \leqq|\mu|(\hat{f}-f)=0$, which completes the proof.

To formulate the main theorem we need some additional requirements on $K$ and on 0 -neighborhoods in $L$.

Definition. Let $U$ and $V$ be subsets in $L . \quad(U, V)$ is called $R$-stable, iff for every $\mu \in U^{0}$ there is $\bar{\mu} \in V^{0} \cap \partial L^{\prime}$ such that $\bar{\mu}-\mu \in$ (lin $N)^{0}$. A subset $U$ in $L$ is called $R$-stable iff $(U, U)$ is $R$-stable.

\section{REMARKS.}

(3.4) Let $U_{1}, U_{2}, V_{1}, V_{2}$ be closed convex 0 -neighborhoods in $L$ such that $\left(U_{1}, V_{1}\right)$ and $\left(U_{2}, V_{2}\right)$ are $R$-stable. Then $\left(U_{1} \cap U_{2}, V_{1} \cap V_{2}\right)$ is $R$-stable.

To prove (3.4) let $\mu \in\left(U_{1} \cap U_{2}\right)^{0}=\operatorname{conv}\left(U_{1}^{0} \cup U_{2}^{0}\right)$. Then $\mu=\lambda_{1} \mu_{1}+$ $\lambda_{2} \mu_{2}, \mu_{1} \in U_{1}^{0}, \mu_{2} \in U_{2}^{0}, \lambda_{1}+\lambda_{2}=1, \lambda_{1}, \lambda_{2} \geqq 0$. By hypothesis there are $\bar{\mu}_{1} \in V_{1}^{0} \cap \partial L^{\prime}$ and $\bar{\mu}_{2} \in V_{2}^{0} \cap \partial L^{\prime}$ such that $\bar{\mu}_{i}-\mu_{i} \in(\operatorname{lin} N)^{0}, i=1,2$. Set $\bar{\mu}=\lambda_{1} \bar{\mu}_{1}+\lambda_{2} \bar{\mu}_{2}$, then $\bar{\mu} \in \partial L^{\prime}, \bar{\mu}-\mu \in(\operatorname{lin} N)^{0}$ and $\bar{\mu} \in \operatorname{conv}\left(V_{1}^{0} \cup V_{2}^{0}\right)=$ $\left(V_{1} \cap V_{2}\right)^{0}$.

(3.5) Suppose $U$ is an $R$-stable closed convex bounded 0 -neighborhood in $L, h \in N \cap \underline{U}$. Then $U-h$ is $R$-stable.

Let $\mu \in(U-h)^{0}$. Then $\operatorname{Re} \mu(h)>-1$ and $\operatorname{Re} \mu(f) \leqq 1+\operatorname{Re} \mu(h)$ for every $f \in U$, hence $\mu \in(1+\operatorname{Re} \mu(h)) U^{0}$. Because $U$ is $R$-stable there is $\bar{\mu} \in \partial L^{\prime} \cap(1+\operatorname{Re} \mu(h)) U^{0}$ such that $\bar{\mu}-\mu \in(\operatorname{lin} N)_{0}$. From $\operatorname{Re} \mu(h)=\operatorname{Re} \bar{\mu}(h)$ we conclude $\operatorname{Re} \bar{\mu}(f) \leqq 1+\operatorname{Re} \bar{\mu}(h)$ for every $f \in U$, hence $\bar{\mu} \in(U-h)^{0}$.

(3.6) There is a handy characterization for $R$-stable admissible 0 -neighborhoods in the case $L$ is a real Banach lattice:

Suppose $U$ is a 0 -neighborhood such that $\mu_{+}\left(\hat{f}_{+}\right)+\mu_{-}\left(\hat{f}_{-}\right) \leqq 1$ 
for all $\mu \in U^{0}, f \in U$, then $U$ is $R$-convex and $(U, U)$ is admissible for every band projection $\pi$ on $L^{\prime}$.

To derive the first assertion, let $\mu_{=}=\mu_{+}-\mu_{-} \in U^{0}$ and select $\mu_{+}, \mu_{-} \in \partial L^{\prime} \cap L_{+}^{\prime}$ such that $\bar{\mu}_{+}>_{R} \mu_{+}, \bar{\mu}_{-}>_{R} \mu_{-}$. Then $\bar{\mu}=\bar{\mu}_{+}-\bar{\mu}_{-} \epsilon$ $\partial L^{\prime}$ and $\bar{\mu}-\mu \in(\operatorname{lin} N)^{0}$ and for every $f \in U$

$$
\bar{\mu}(f) \leqq \bar{\mu}_{+}\left(f_{+}\right)+\bar{\mu}_{-}\left(f_{-}\right) \leqq \bar{\mu}_{+}\left(\hat{f}_{+}\right)+\bar{\mu}_{-}\left(\hat{f}_{-}\right) \leqq \mu_{+}\left(\hat{f}_{+}\right)+\bar{\mu}_{-}\left(\hat{f}_{-}\right) \leqq 1,
$$

hence $\bar{\mu} \in U^{0}$. Secondly assume $\pi$ is a band projection on $L^{\prime}$. Then $\pi(\mu)(f)=\pi\left(\mu_{+}\right)(f)-\pi\left(\mu_{-}\right)(f) \leqq \pi\left(\mu_{+}\right)\left(f_{+}\right)+\pi\left(\mu_{-}\right)\left(f_{-}\right) \leqq \mu_{+}\left(\hat{f}_{+}\right)+\mu_{-}\left(\hat{f}_{-}\right) \leqq 1$, hence $\pi \mu \in U^{0}$.

Now we state

THEOREM 3. Let $L$ be a real or complex Banach lattice, $M a$ real linear subspace of $L, N$ a closed convex cone in $M, R$ a supstable convex cone in $L_{0}$ (the underlying real Banach lattice of $L$ ) such that (3.1), (3.2), and (3.3) hold. Suppose $K$ is an $R$-stable ideal in $L, p: L \rightarrow L / K$ the canonical projection, $\pi: L^{\prime} \rightarrow K^{0}$ the band projection from $L^{\prime}$ onto $K^{0}$. For the following assertions

(a) For each triple $(U, W, V)$ of closed convex bounded 0-neighborhoods in $L$ such that $(U, W)$ is $R$-stable and $(W, V)$ is admissible (with respect to $\pi$ )

$$
p(N \cap U) \supset p(M) \cap \underline{p(V) .}
$$

(b) For every strongly admissible R-stable closed convex bounded 0-neighborhood $U$ in $L$

$$
p(N \cap U) \supset p(M) \cap \overline{p(U)} .
$$

(c) $\pi\left(\partial L^{\prime} \cap N^{0}\right) \subset M^{0}$ and $K^{0} \cap(\operatorname{lin} N)^{0} \subset M^{0}$.

(a) implies (b), and (c) and (a) are equivalent.

Proof.

(a) $\Rightarrow$ (b). For every $h \in N \cap U \quad U_{h}=U \cap(1 / \lambda(h))(U-h)$ is $R$ stable (Remarks (3.4) and (3.5)) and $\left(U_{k}, U\right)$ is admissible, (b) then is a sequence of implication $(\mathrm{e}) \Rightarrow(\mathrm{b})$ in Theorem 1 .

(c) $\Rightarrow$ (a). To apply Theorem $1,(\mathrm{~d}) \Rightarrow(\mathrm{a})$, we construct $\varphi: L^{\prime} \rightarrow K^{0}$ as follows: Let $\mu \in L^{\prime}$ and $\lambda=\inf \left\{\rho \in \boldsymbol{R}_{+} \mid \mu \in \rho U^{0}\right\}$. There is $\bar{\mu} \in \lambda W^{0} \cap \partial L^{\prime}$, such that $\bar{\mu}-\mu \in(\operatorname{lin} N)^{0}$. Define $\varphi(\mu)=\pi(\bar{\mu}) \in \lambda V^{0}$. Conditions (d1), (d2), (d3) hold.

(d1): Let $\mu \in K^{0}$. Because of the $R$-stability of $K$ there is $\bar{\nu} \in K^{0} \cap \partial L^{\prime}$ such that $\mu-\bar{\nu} \in(\operatorname{lin} N)^{0}$, hence $\mu-\bar{\nu} \in K^{0} \cap(\operatorname{lin} N)^{0} \subset M^{0}$ by assumption (c). On the other hand $\bar{\mu}$ in the construction of $\varphi(\mu)$ 
was selected such that $\bar{\mu}-\mu \in(\operatorname{lin} N)^{0}$, hence $\bar{\mu}-\bar{\nu} \in \partial L^{\prime} \cap(\operatorname{lin} N)^{0} \subset$ $\partial L^{\prime} \cap N^{0}$ and again by (c) we conclude $\pi(\bar{\mu}-\bar{\nu})=\pi(\bar{\mu})-\bar{\nu}=\varphi(\mu)-$ $\bar{\nu} \in M^{0}$, hence, $\varphi(\mu)-\mu \in M^{0}$. (d2) is obvious, because $\mu \in U^{0}$ implies $\varphi(\mu) \in V^{0}$. To verify (d3) let $\mu, \nu \in L^{\prime}$ such that $(\mu-\nu) \in N^{0}$. Then $\bar{\mu}-\bar{\nu} \in \partial L^{\prime} \cap N^{0}$, hence by (c) $\pi(\bar{\mu}-\bar{\nu})=\varphi(\mu)-\varphi(\nu) \in M^{0}$.

(a) $\Rightarrow$ (c). Clearly $K^{0} \cap(\operatorname{lin} N)^{0} \subset M^{0}$ is a necessary condition for (a) because (a) implies $p(N)=p(M)$. To prove the other inclusion let $\mu \in \partial L^{\prime} \cap N^{0}, f \in M$. To show $\operatorname{Re} \pi(\mu)(f) \leqq 1$ we construct a proper triple of neighborhoods $U_{\varepsilon}, W_{\varepsilon}, V$ : Define

$$
U_{\varepsilon}=E \cap\{h \in L||(I-\pi)(\mu)(h) \mid \leqq \varepsilon\}
$$

( $E$ denotes the unit ball in $L$ ). By assumption (3.3) for $R$ there is a constant $r>0$ such that $r E_{L_{0}} \subset\left(E_{L_{0}} \cap R\right) . \quad\left(E_{L_{0}}\right.$ is the unit ball in $L_{0}$.)

Let $W_{\varepsilon}=(r / 4) E \cap\{h \in L||(I-\pi)(\mu)(h) \mid \leqq \varepsilon\}, \quad V=(r / 4) E . \quad$ The pair $\left(W_{\varepsilon}, V\right)$ is admissible (cf. the proof $(\mathrm{a}) \Rightarrow(\mathrm{c})$ in Theorem 2). We shall prove now $\left(U_{\varepsilon}, W_{\varepsilon}\right)$ is $R$-stable. Let $\mu \in E^{0}$, then there is a decomposition $\mu=\mu_{1}-\mu_{2}+i\left(\mu_{3}-\mu_{4}\right)$ such that $\mu_{i} \in E^{0} \cap L_{+}^{\prime}$. Let $\bar{\mu}_{i}$ be $R$-maximal in $L_{+}^{\prime}$ such that $\bar{\mu}_{i}>_{R} \mu_{i}$. Suppose $f \in r E_{L_{0}}$ then there is $h \in E_{L_{0}} \cap-R$ such that $h \geqq f$, hence $\bar{\mu}_{i}(f) \leqq \bar{\mu}_{i}(h) \leqq \mu_{i}(h) \leqq 1$, hence $\bar{\mu}_{i} \in\left(r E_{L_{0}}\right)^{0}$, and for $f \in r E$ we conclude $f=f_{1}+i f_{2}, f_{1}, f_{2} \in r E_{L_{0}}$, hence $\operatorname{Re} \bar{\mu}_{i}(f)=\bar{\mu}_{i}\left(f_{1}\right) \leqq 1$, and $\bar{\mu}_{i} \in(r E)^{0}$. Thus

$$
\bar{\mu}=\bar{\mu}_{1}-\bar{\mu}_{2}+i\left(\bar{\mu}_{3}-\bar{\mu}_{4}\right) \in 4(r E)^{0}=\left(\frac{r}{4} E\right)^{0},
$$

and $(E,(r / 4) E)$ is $R$-stable. Because of Remark (3.4) all left to show now is $R$-stability of the set $\{h \in L|(I-\pi)(\mu)(h)|<\varepsilon\}$. But this is obvious because $\mu \in \partial L^{\prime}$ implies $(I-\pi)(\mu) \in \partial L^{\prime}$ (cf. Lemma 3) and $\{\cdots\}^{0}=\{\lambda(I-\pi)(\mu)|| \lambda \mid \leqq 1\} \subset \partial L^{\prime}$. Therefore $\left(U_{\varepsilon}, W_{\varepsilon}\right)$ is $R$-stable, and to complete the proof we adapt the conclusion in (a) $\Rightarrow$ (c) in Theorem 2.

4. The case $L=C(X)$. There are some interesting applications of Theorem 3 to the case $L=C(X)$, where $X$ is a compact Hausdorff space. With proper choice of the parameter $R$ then quite a few generalizations of well-known results about dominated extensions of continuous functions are at hand. We have to distinguish the cases $L=C_{R}(X)$ (real valued continuous functions on $X$ ) and the complex case $L=C_{c}(X)$. The latter one requires more sophisticated techniques to stady $R$-stable neighborhoods, corresponding to Hustad's [14] method to derive a norm preserving: complex Choquet theorem. We apply a generalization of his result [18].

Throughout the chapter suppose $X$ is a compact Hausdorff space, 
$L=C_{R}(X)$ (resp. $\left.L=C_{c}(X)\right)$ provided with the supremum norm. Let $K$ be the closed ideal in $C(X)$ of all functions vanishing on the compact subset $Y \subset X . \quad L^{\prime}$ then is the space of all real (resp. complex) valued Borel measures on $X, \pi: L^{\prime} \rightarrow K^{0}$ the usual restriction to the subset $Y$.

To define strongly admissible 0 -neighborhoods in $L$ let $\gamma=$ $\{z \in \boldsymbol{C}|| z \mid=1\}, \rho: X \times \gamma \rightarrow \boldsymbol{R}_{+}$a lower semicontinuous bounded strictly positive function and

$$
U=\left\{f \in C_{C}(X) \mid \operatorname{Re}(z f(x)) \leqq \rho(x, z) \text { for all } x \in X, z \in \gamma\right\} .
$$

To see that $U$ is a strongly admissible 0-neighborhood in $L=C_{c}(X)$ with respect to the restriction map $\pi$, let $f, g \in U, \mu \in U^{0}, \chi_{Y}$ the characteristic function of $Y$. We shall prove first that

$$
\operatorname{Re} \mu\left(f \chi_{Y}+g\left(1-\chi_{Y}\right)\right) \leqq 1 .
$$

Given $\varepsilon>0$ there is a compact subset $K \in X \backslash Y$ such that

$$
|\mu|(X \mid(Y \cup K))<\varepsilon .
$$

Let $x \in X \backslash(Y \cup K)$. Urysohn's lemma guanties the existence of continuous functions $\chi_{x}$ and $\phi_{x}$ such that $0 \leqq \phi_{x}, \psi_{x} \leqq 1$ and

$$
\begin{aligned}
\left.\psi_{x}\right|_{K \cup\{x\}} & =0, & & \left.\psi_{x}\right|_{Y}=1 \\
\left.\phi_{x}\right|_{Y \cup\{x\}} & =0, & & \left.\phi_{x}\right|_{K}=1 .
\end{aligned}
$$

Let $\alpha<1$ and $\bar{f}=\alpha f, \bar{g}=\alpha g$, and

$$
G_{x}=\left\{(x, z) \in X \times \gamma \mid \operatorname{Re}\left(z\left(\phi_{x} \bar{f}+\psi_{x} \bar{g}\right)\right)<\rho(x, z)\right\}
$$

then $Y \times \gamma, K \times \gamma,\{x\} \times \gamma$ all are subsets of $G_{x}$, which is open in $X \times \gamma$, because $\rho$ is lower semicontinuous. $\operatorname{Re} z\left(\dot{\phi}_{x} \bar{f}+\psi_{x} \bar{g}\right)$ is continuous on $X \times \gamma$. Thus $\mathrm{U}_{x} G_{x}=X \times \gamma$, which is compact, and there are $x_{1}, x_{2}, \cdots, x_{n} \in X \backslash(Y \cup K)$ such that $\bigcup_{i=1}^{n} G_{x_{i}}=X \times \gamma$. Let $\psi=$ $\inf \psi_{x_{i}}, \phi=\inf \phi_{x_{i}}$. Thus $h=\phi \bar{f}+\psi \bar{g} \in U,\left.h\right|_{Y}=\bar{f},\left.h\right|_{K}=\bar{g}$, and

and

$$
\left|\mu(h)-\mu\left(\bar{f} \chi_{Y}+\bar{g}\left(1-\chi_{Y}\right)\right)\right| \leqq \varepsilon(\|\bar{f}\|+\|\bar{g}\|),
$$

$$
\left|\mu(h)-\mu\left(f \chi_{Y}+g\left(1-\chi_{Y}\right)\right)\right| \leqq \varepsilon(\|f\|+\|g\|)+\|\mu\|(1-\alpha) .
$$

Because $\|f\|,\|g\|,\|\mu\|$ are bounded, $\varepsilon>0$ and $\alpha<1$ arbitrary, and $h \in U$ we conclude (4.1).

Now set $\lambda=\sup \{\operatorname{Re} \pi(\mu)(f) \mid f \in U\}, \delta=\sup \{\operatorname{Re}(1-\pi)(\mu)(f) \mid f \in U\}$. Then $\lambda, \delta \geqq 0, \lambda+\delta \leqq 1$ by $(4.1)$, and $\pi(\mu) \in \lambda U^{0},(1-\pi)(\mu) \in \delta U^{0}$, hence $\pi(\mu) \in \lambda \pi\left(U^{0}\right),(1-\pi)(\mu) \in \delta(1-\pi)\left(U^{0}\right)$, and

$$
\begin{aligned}
\mu=\pi(\mu)+(1-\pi)(\mu) & \in \operatorname{conv}\left(\pi\left(U^{0}\right) \cup(1-\pi)\left(U^{0}\right)\right) \\
U & \subset \operatorname{conv}\left(\pi\left(U^{0}\right) \cup(1-\pi)\left(U^{0}\right)\right) .
\end{aligned}
$$


The converse is obvious, since $\mu \in U^{0}, f \in U$ implies by $(4.1) \pi(\mu)(f)=$ $\mu\left(f \chi_{Y}\right) \leqq 1$, hence $\pi \mu \in U^{0}$.

As a first corollary of Theorem 3 choosing $R=C_{R}(X)$ we now prove a Rudin-Carleson theorem, which generalizes Gamelin's [10] version by requiring $N$ only to be a convex cone:

CoRollary 3.1. Let $Y$ be a closed subset of the compact Hausdorff space $X, M$ a real linear subspace of $C_{c}(X), N$ a closed convex cone in $M$. Then the following conditions are equivalent:

(a) For every 0-neighborhood $U$ in $C_{c}(X)$ defined by a strictly positive bounded lower semicontinuous function $\rho: X \times \gamma \rightarrow \boldsymbol{R}_{+}$(as above) and every $f \in M$ such that $f_{\mid Y} \in U_{\mid Y}$ (restrictions to the subset $Y$ ) there is a function $g \in N \cap U$ such that $g_{\mid Y}=f_{\mid Y}$.

(b) For every complex Borel measure $\mu$ on $X \mu \in N^{0}$ implies $\mu_{\mid Y} \in M^{0}$.

\section{Proof.}

(b) $\Rightarrow(a)$ is an immediate consequence of $(c) \Rightarrow(b)$ in Theorem 3 . To prove the converse suppose $\mu \in N^{0}, h \in M$ such that $h \neq 0$. Define $U$ by $\rho(x, \varphi)=\|h\|$ if $x \in G$ and $\rho(x, \varphi)=\varepsilon$ else, where $G$ is an open neighborhood of $Y$. Clearly $h_{\mid Y} \in U_{\mid Y}$ and by assumption there is $g \in N \cap U$ such that $h_{\mid Y}=g_{Y}^{\prime}$, hence

$$
\begin{aligned}
0 \geqq \operatorname{Re} \mu(g) & =\operatorname{Re} \mu_{\mid Y}(g)+\operatorname{Re} \mu_{\mid G-Y}(g)+\operatorname{Re} \mu_{\mid X-G}(g) \\
& \geqq \operatorname{Re} \mu_{\mid Y}(h)-h|\mu|(G-Y)-\varepsilon|\mu|(X-G),
\end{aligned}
$$

and because $G$ and $\varepsilon$ were arbitrary and $\mu$ is regular $0 \geqq \operatorname{Re} \mu_{\mid Y}(h)$, hence $\mu_{\mid Y} \in M^{0}$.

We are going to state now a corollary, which implies and generalizes results by Björk [10], Alfsen-Hirsberg [2], and T.B. Andersen [3]. Recall that the Choquet boundary of $R \partial_{R} X$ is defined to be the subset of all $x \in X$ such that the Dirac measure $\varepsilon_{x}$ is maximal in the " $<_{R}$ " ordering. Every "boundary measure" $\mu \in \partial L^{\prime}$ on $X$ is known to vanish on every Baire set disjoint from the Choquet boundary (cf. [1] or [14]). For a linear subspace $N$ in $C_{c}(X)$, which separates the points of $X$ and contains the constants, we say $\partial_{N} X=\partial_{R} X$, where $R$ is the sup-stable cone in $C_{R}(X)$ generated by the real parts of $N$.

Note that in the real case the $R$-stability of a given neighborhood $U$ is relatively easy to be checked, whereas in the complex case the arguments turn out to be much more complicated. Hustad [14] (along with Hirsberg's [13] interpretation) proves the $R$-stability of the unit ball in $C_{c}(X)$. We shall apply a generalization of his result given in [18]: 
Suppose $U$ is defined by a strictly positive l.s.c. function $\rho: X \times \gamma \rightarrow R \cup\{\infty\}$

$$
U=\left\{f \in C_{C} \mid \operatorname{Re}(z f(x)) \leqq \rho(x, z), \text { for all } x \in X, z \in \gamma\right\}
$$

and for every $z \in \gamma$, the function

$$
\rho_{z}: X \rightarrow \boldsymbol{R} \cup\{\infty\}, \quad \rho_{z}(x)=\rho(z, x)
$$

is $R$-superharmonic, i.e., $\rho_{z}(x) \geqq \mu\left(\rho_{z}\right)$ for all $x \in X$ and $\mu>_{R} \varepsilon_{x}$ (Dirac measure in $x$ ). Then $U$ is $R$-stable.

Corollary 3.2. Let $X$ be a compact Hausdorff space, $M$ a real linear subspace in $C_{c}(X)$ (resp. $C_{R}(X)$ ), $N$ a closed convex cone in $M$, which separates the points of $X$ and contains the constant functions, $R$ a sup-stable convex cone in $C_{R}(X)$ which contains the real parts of all functions in lin $N$.

Suppose $Y$ is a compact subset of $X$ such that

(1) for every measure $\mu$ supported by $Y$ there is a boundary measure $\bar{\mu}$ supported by $Y$ such that $\bar{\mu}-\mu \in(\operatorname{lin} N)^{0}$.

(2) for every complex boundary measure $\mu \in N^{0}$ implies $\mu_{\mid Y} \in M^{0}$.

(3) lin $N_{\mid Y}$ is dense in $M_{\mid Y}$.

Suppose $U$ is a 0-neighborhood in $C_{c}(X)$ defined by a strictly positive bounded l.s.c. function $\rho: X \times \gamma \rightarrow R$, such that $\rho_{z}: X \rightarrow R$ is $R$-superharmonic for every $z \in \gamma$.

Then for every $f \in M$ such that $f_{\mid Y} \in U_{\mid Y}$ there is $g \in N \cap U$ such that $f_{\mid Y}=g_{\mid Y}$.

With the above notations and remarks this follows directly from Theorem 3. If $Y$ is a subset of $\partial_{1 \text { in } N} X$ condition (1) is obviously true, (2) implies (3), so Corollary (3.2) generalizes Björk's [10] result and the main theorem in the Alfsen-Hirsberg paper [2]. To derive a complex version of T.B. Andersen [3] extension theorem about continuous affine functions on split-faces let $Y$ be a closed split-face in the compact convex set $X, N=A(X)$ the space of all continuous (complex) affine functions on $X, M$ the subspace of $C_{c}(X)$ such that all function in $M_{\mid Y}$ are affine on $X$. Conditions (1) and (3) then are obvious, because $Y$ is a face and because $A(X)_{\mid Y}$ is dense in $A(Y)$. (2) is known to be a characterization for split-faces (cf. [1], Theorem II.6.12).

Note that in the real case $\rho$ reduces to two strictly positive bounded 1.s.c. functions $f_{0}, f_{U}: X \rightarrow R_{+}$defining $U$ by

$$
U=\left\{f \in C_{R}(X) \mid-f_{U} \leqq f \leqq f_{0}\right\}
$$

$U$ is $R$-stable if both $f_{U}$ and $f_{0}$ are $R$-superharmonic. 
Another obvious consequence of our main result is Alfsen's Theorem II. 4.5 [1].

COROLLARY 3.3. Let $Y$ be the topological closure of the set of extreme points $\partial_{e} X$ of the compact convex set $X, f: \partial_{e} X \rightarrow R$ a continuous function. Then $f$ can be extended to a function in $A(X)$ iff the following two conditions are satisfied:

(1) $\hat{f}$ and $\check{f}$ coincide on $\overline{\partial_{e} K}$ ( $\hat{f}$ is defined to be $\inf \{g \in A(X) \mid g \geqq f\}$ ).

(2) The common restriction of $\hat{f}$ and $\tilde{f}$ to $\overline{\partial_{e} X}$ is annihilated by every $\mu \in \partial L^{\prime} \cap A(X)^{0}$.

To prove this set $N=A(X), M=A+\boldsymbol{R} \widetilde{f}$, where $\widetilde{f}$ is any continuous extension of $f$ on $X, U$ the unit ball in $C_{R}(X)$. With the same choice of $K$ and $R$ as before the assertion is obvious.

Finally we are going to derive a corollary of the type of Bauer's classical theorem on the abstract Dirichlet-problem (cf. [8], [1] Theorem II. 4.3, [17]).

Corollary 3.4. Let $N$ be a closed convex cone in $C(X)\left(C_{c}(X)\right.$ resp. $C_{R}(X)$ ), where $X$ is a compact Hausdorff space, which separates the points of $X$ and contains the constant functions. Set $Y=\overline{\partial_{1 \text { in } N} X}$ and $R$ the sup-stable convex cone generated by lin $N$. Then $N_{\mid Y}=C(Y)$ if and only if $N^{0} \cap \partial L^{\prime}=\{0\}$ and $\partial_{1 \mathrm{in} N} X=Y$.

To prove this set $M=C(X) . \quad K=\left\{f \in C(X) \mid f_{\mid Y}=0\right\}$ is $R$-stable as well as the unit ball $U$ in $C_{R}(X)$. All left to show is $K^{0} \cap$ $(\text { lin } N)^{0} \subset M^{0}$. But this is obvious because $\mu \in K^{0} \cap(\operatorname{lin} N)^{0}$ implies $\mu \in \partial L^{\prime}\left(K^{0}\right.$ is the set of all measures carried by $Y=\partial_{\text {in }_{N}} X$, hence the set of all boundary measures), therefore $\mu \in \partial L^{\prime} \cap N^{0}=\{0\}$.

\section{REFERENCES}

1. E. M. Alfsen, Compact convex sets and boundary integrals, Ergebnisse der Mathematik, 57, Springer-Verlag, Germany.

2. E. M. Alfsen and B. Hirsberg, On dominated extensions in linear subspaces in $C_{c}(X)$, Pacific J. Math., 36 (1971), 567-584.

3. T. B. Andersen, On dominated extension of continuous affine functions on split faces, Math. Scand., 29 (1971), 298-306.

4. T. Ando, Closed range theorems for convex sets and linear liftings, Pacific J. Math., 44 (1973), 393-409.

5. L. Asimow, Extensions of continuous affine functions, Pacific J. Math., 35 (1970), 11-21.

6. Complementary cones in dual Banach spaces, Illinois J. Math., 18 No. 4, (1974).

7. - Best approximation by ganges on a Banach space, to appear. 
8. H. Bauer, Silovscher Rand und Dirichletsches Problem, Ann. Inst. Fourier, 11 (1961), 89-136.

9. Bishop, A general Rudin-Carleson theorem, Proc. Amer. Math. Soc., 13 (1962), 140-143. 10. J. E. Björk, Interpolation on closed subspaces of $C(X)$, to appear.

11. E. Briem, Interpolation in subspaces of $C(X)$, J. Functional Analysis, 12 (1973), $1-12$.

12. T. W. Gamelin, Uniform Algebras, Prentice-Hall, Englewood Cliffs, N.J., 1969.

13. B. Hirsberg, Représentations intégrales des formes linéaires complexes, C.R. Acad. Sci. Paris, Ser. A 274 (1972), 1222-1224.

14. O. Hustad, A norm preserving complex Choquet theorem, Math. Scand., 29 (1971), 272-278.

15. R. Kaufmann, Interpolation of additive functionals, Studia Math., 27 (1969), 269-272.

16. R. R. Phelps, Lectures on Choquet's Theorem, Van Nostrand, New York, 1966.

17. W. Roth, Uniform approximation by elements of a cone of real valued functions, Pacific J. Math., 60 (1975), 209-215.

18. A stability theorem for the Choquet ordering in $C_{C}(X)$, to appear.

19. H. H. Schaefer, Banach lattices and Positive Operators, Die Grundlehren der mathematischen Wissenschaften in Einzeldarstellungen, 215, Springer-Verlag, Germany. 20 . , Topological Vector Spaces, GTM 3, Springer-Verlag.

Received February 15, 1974 and in revised form June 2, 1977.

TEChNISChe HoChSChUle

D61 DARMSTADT

West Germany 



\section{PACIFIC JOURNAL OF MATHEMATICS}

EDITORS

RICHARD ARENS (Managing Editor)

University of California

Los Angeles, California 90024

C. W. Curtis

University of Oregon

Eugene, OR 97403

C. C. MOORE

J. DUGUNDJI

Department of Mathematics University of Southern Californa Los Angeles, California 90007

R. Finn AND J. Milgram Stanford University Stanford, California 94305

University of California

Berkeley, CA 94720

\section{ASSOCIATE EDITORS}

E. F. BeCKENBACH

B. H. NeUMANN

F. WOLF

K. YosHida

\section{SUPPORTING INSTITUTIONS}

UNIVERSITY OF BRITISH COLUMBIA UNIVERSITY OF SOUTHERN CALIFORNIA CALIFORNIA INSTITUTE OF TECHNOLOGY UNIVERSITY OF CALIFORNIA MONTANA STATE UNIVERSITY UNIVERSITY OF NEVADA, RENO STANFORD UNIVERSITY UNIVERSITY OF TOKYO UNIVERSITY OF UTAH NEW MEXICO STATE UNIVERSITY WASHINGTON STATE UNIVERSITY OREGON STATE UNIVERSITY UNIVERSITY OF WASHINGTON UNIVERSITY OF OREGON OSAKA UNIVERSITY 


\section{Pacific Journal of Mathematics \\ Vol. 73, No. 1 \\ March, 1977}

Thomas Robert Berger, Hall-Higman type theorems. $V \ldots \ldots \ldots \ldots \ldots \ldots \ldots$

Frank Peter Anthony Cass and Billy E. Rhoades, Mercerian theorems via

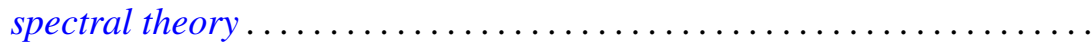

Morris Leroy Eaton and Michael David Perlman, Generating $\mathrm{O}(n)$ with

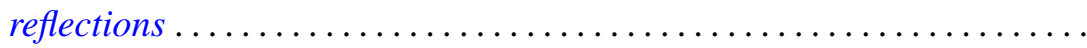

Frank John Forelli, Jr., A necessary condition on the extreme points of a

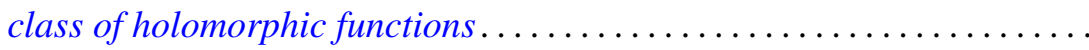

Melvin F. Janowitz, Complemented congruences on complemented

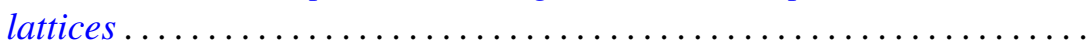

Maria M. Klawe, Semidirect product of semigroups in relation to amenability, cancellation properties, and strong $F \phi$ lner conditions....

Theodore Willis Laetsch, Normal cones, barrier cones, and the "spherical image" of convex surfaces in locally convex spaces ................

Chao-Chu Liang, Involutions fixing codimension two knots.............

Joyce Longman, On generalizations of alternative algebras .............

Giancarlo Mauceri, Square integrable representations and the Fourier

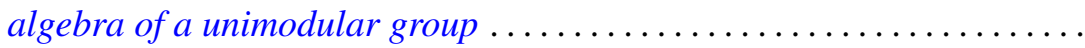

J. Marshall Osborn, Lie algebras with descending chain condition...

John Robert Quine, Jr., Tangent winding numbers and branched

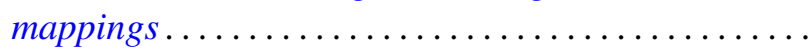

Louis Jackson Ratliff, Jr. and David Eugene Rush, Notes on ideal covers and associated primes .

H. B. Reiter and N. Stavrakas, On the compactness of the hyperspace of faces.

Walter Roth, A general Rudin-Carlson theorem in Banach-spaces ..

Mark Andrew Smith, Products of Banach spaces that are uniformly rotund in every direction.

Roger R. Smith, The R-Borel structure on a Choquet simplex ...

Gerald Stoller, The convergence-preserving rearrangements of real infinite series. ...

Graham H. Toomer, Generalized homotopy excision theorems modulo a Serre class of nilpotent groups...

Norris Freeman Weaver, Dehn's construction and the Poincaré conjecture....

Steven Howard Weintraub, Topological realization of equivariant intersection forms... 